International Journal of Pure and Applied Mathematics

Volume 99 No. $4 \quad 2015,411-437$

ISSN: 1311-8080 (printed version); ISSN: 1314-3395 (on-line version)

url: http://www.ijpam.eu

doi: http://dx.doi.org/10.12732/ijpam.v99i4.3

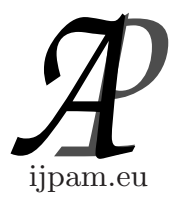

\title{
MATHEMATICAL ANALYSIS OF THE DYNAMICS OF MALARIA DISEASE TRANSMISSION MODEL
}

\author{
E.A. Bakare ${ }^{1}$, C.R. Nwozo ${ }^{2}$ \\ ${ }^{1}$ Department of Mathematics \\ Federal University Oye Ekiti \\ Ekiti State, NIGERIA \\ ${ }^{2}$ Department of Mathematics \\ University of Ibadan \\ Ibadan, NIGERIA
}

\begin{abstract}
We formulate a deterministic model for the transmission dynamics of malaria parasite in mosquito and human. The model, which allows for the transmission of the parasite, has a global asymptotic stable disease-free if a certain epidemiological threshold called the reproductive number is less than unity. We realized that the model has a unique academic equilibrium whenever this threshold exceeds unit. We proved that, using a Lyapunov function, that this endemic equilibrium is globally asymptotically stable whenever the basic reproduction number is greater than unity. We also carried out numerical simulations to support our analytical results.
\end{abstract}

AMS Subject Classification: 92B05, 92D25, 92D30, 93D05, 34K20, 34K25 Key Words: basic reproduction number, global asymptotic stability, diseasefree-equilibrium, endemic equilibrium, Lyapunov function

Received: May 1, 2014

(c) 2015 Academic Publications, Ltd. url: www.acadpubl.eu

${ }^{\S}$ Correspondence author 


\section{Introduction}

Malaria is an infectious disease caused by a parasite that is transmitted by the bite of a female Anopheles mosquito.Malaria is one of the world's most prevalent vector-borne disease. Despite decades of global eradication and control efforts, the disease is re-emerging in areas where control efforts were once effective and emerging in areas thought free of the disease[28,17,22,14,23].As reported in World Health Organisation(WHO) fact sheet(2009), malaria a life threatening disease caused by parasites that are transmitted to people through the bites of infected mosquitoes, which resulted in the death of a child from malaria every 30secs[16]. An estimated 40percent of the world's population live in malaria endemic areas. The disease kills about 1 to 3 million people a year, 75percent of whom are African children. The incidence of malaria has been growing recently due to increasing parasite drug-resistance and mosquito insecticide-resistance[4]. Therefore, we find it useful and important to study and understand the important parameters in the dynamics of transmission of the disease in order to help in the effective control strategies.

Mathematical models for transmission dynamics of malaria are useful in providing a better knowledge of the disease, to plan for the future and consider appropriate control measures. Models have played great roles in the development of the epidemiology of the disease. The study on malaria using mathematical modeling originated from the works of Ross [21]. According to Ross, if the mosquito population can be reduced to below a certain threshold then malaria can be eradicated. MacDonald did some modification to the model and included superinfection $[15,13]$. He showed that reducing the number of mosquitoes have little effect on epidemiology of malaria in areas of intense transmission. Dietz et al $[8,13]$ added two classes of humans in their mathematical model, namely those with low recovery rate (more infections, greater susceptibility) and high recovery rate (less infections, less susceptibility). Compartmental models of malaria and differential equations are constructed to model the disease $[26,7,4,9,13,20]$. Chitnis et al [4] did a bifurcation analysis of a malaria model. Malaria transmission model which incorporate immunity in the human population had been studied $[26,7,9,13]$. Epidemiological models on the spread of anti-malarial resistance were also constructed [12].Anderson and May[2]formulated a malaria model with the assumption that acquired immunity in malaria is independent of exposure duration. They also examined different control measures and role of transmission rate on the disease prevalence. The works of Ross and Macdonald were further extended by [20] with the popular generalized SEIR malaria model, which includes both the human and mosquito interactions. Koella and 
Anita [12] included a latent class for mosquitoes. They considered different intervention to reduce the spread of resistance and studied the sensitivity of their results to the parameters. Hyun[9] studied a malaria transmission model for different levels of acquired immunity and temperature dependent parameters, relating also to global warming and local socioeconomic conditions. Isao et al[10] investigated the combined use of insecticides sprays and zooprophylaxis as a strategy for malaria control. Tumwiine et al.[25] used an SIS and SI model in the human hosts and mosquito vectors respectively, for the study of malaria epidemic that lasts for a short period in which birth and immunity to disease were ignored. They observed that the system was in equilibrium only at the point of extinction, that was neither stable or unstable. However, some important results were revealed numerically. Also, Tumwiine et al[26] proposed a model that tracks the dynamics of malaria in the human host and mosquito vector. Their model incorporates some infected humans that recover from infection and immune humans after loss of immunity to the disease to join the susceptible class again. The model was later reformulated in terms of the proportions of the classes of the respective population. They observed that since malaria induced immunity wanes overtime and there are no effective vaccines against it at the moment,the available tools can be used for its control. Chiyaka et al.[8] proposed a deterministic model with two latent periods in the host and vector populations to assess the impact of personal protection, treatment and possible vaccination strategies on the transmission dynamics of malaria. They also considered treatment and spread of drug resistance in an epidemic population[9]. Jia[11] developed and examined a compartmental mathematical model for malaria transmission that includes incubation periods for both infected humans and mosquitoes.

Tumwiine et al[24] developed a mathematical model for the dynamics of malaria with a varying population for which new individuals are recruited through immigration and births. In the model they assume that non-immune travellers move to endemic regions with sprays, snear themselves with jelly that is repellant to mosquitoes on arrival infants received full treatment doses at intervals even when they are not sick from malaria(commonly referred to as intermittent preventive therapy). They also introduce more features that describe the dynamics of the disease for the control strategies that protect the vulnerable groups. They analyze the model and the equilibrium point to establish their local and global stability. Tumwiine et al.[23] developed a host-vector mathematical model for the spread of malaria that incorporates recruitment of human population through a constant immigration, with a fraction of infective immigrants. They observed that when the fraction of infective 
immigrants approaches a small value, there is sharp threshold for which the disease can be reduced in the community. White et al[27] proposed a model to compare distinct interventions, such as indoor residual spraying, long lasting insecticide treated bedNets, larvicides and pupacides and their combinations in malaria endemic settings in sub-Sahara Africa. They argued that the selection of combinations of interventions used in different stages in the vector's life cycle, would strongly reduced Anopheles gambiae mosquito densities.Aguas et al[1] compare two mathematical models of transmission for P.vivax and P.falciparum parasites their work suggested that artemisinin-based combination therapy combined with a hypnozoite killing drug, would eliminate both species. Nevertheless, P.vivax 's ability to relapse accelerated the acquisition of presenile clinical immunity. This parasite transmission persisted in areas of low mosquito abundance and was robust to drug administration initiatives due to relapse.Nevertheless, P.vivax was less lethal than P.falciparum.Li-ming Cai et al.[3] described a deterministic model with variable human population for the transmission dynamics of malaria disease, which allow transmission by the recovered humans, is first developed and rigorously analyzed. They observed that in some regions where malaria is inducing the varying total populations, it is difficult to control malaria is inducing the varying total populations, it is difficult to control malaria due to the occurrence of backward bifurcation phenomenon. Mukandavire et al [18]proposed and examined a deterministic model for the co-infection of HIV and malaria in a community. Mwasa and Tchuenche[19] investigated a mathematical model that captures the dynamics of cholera transmission to study the impact of public health educational campaign, vaccination and treatment in controlling the disease.Nakul Chitnis et al[4] investigate an ordinary differential equation mathematical model for spread of malaria in human and mosquito populations. They observed that in the absence of disease-induced death, we prove that the transcritical bifurcation at $R_{0}=1$ is supercritical(forward) and numerical simulations shows that for larger values of the disease-induced death rate, a subcritical(backward) bifurcation is possible at $R_{0}=1$.

Our goal in this work is to investigate and understand the effect of logistic growth model due to the transmission of malaria in a fully homogeneous population and to inspect the influence of the embedded parameters in the dynamic of the malaria disease model.In this paper, we present a malaria transmission model formulation in section 2 , where the general mathematical framework, notations and model equations including the $S_{h} E_{h} I_{h} R_{h} S_{h}+S_{v} E_{v} I_{v}$ model were analyzed. In the third and fourth section the basic reproduction number were derived and the stability criteria for the disease free and endemic equilibrium 
were discussed respectively, the fifth section is concern with the numerical simulation results, while the last section deals with summary and conclusions.

\section{Model Formulation}

We consider a standard $S_{h} E_{h} I_{h} R_{h} S_{h}+S_{v} E_{v} I_{v}$ with bilinear incidence and variable total human population. Suppose $S_{h}$ represents the number of susceptible humans, $E_{h}$ represents the number of exposed humans, $I_{h}$ represents the number of individual who are infected and infectious, and $R_{h}$ represents the number of individuals who recovered from the malaria disease for a while. The model subdivides the total vector population at time $t$ denoted by $N_{v}(t)$, into susceptible vector $\left(S_{v}(t)\right)$, into exposed vector $\left(E_{v}(t)\right)$, Infected vectors $\left(I_{v}(t)\right)$, so that

$$
N_{v}(t)=S_{v}(t)+E_{v}+I_{v}(t) .
$$

Similarly, the total human population at time $t$, denoted by $N_{h}(t)$ is subdivided into Susceptible humans $\left(S_{h}(t)\right)$, Exposed humans $E_{h}$, Infected humans $\left(I_{h}(t)\right)$, Recovered humans $\left(R_{h}(t)\right)$. Therefore,

$$
N_{h}(t)=S_{h}(t)+E_{h}(t)+I_{h}(t)+R_{h}(t)
$$

. The population of susceptible humans is generated by birth (recruitment) of humans (at a per capita rate $\lambda_{h} N_{h}$ ). It is reduced by infection, following contacts with infected vectors (at a rate $\frac{\beta_{1} S_{h} I_{v}}{N_{v}}$ ) where $\beta_{1}$ is the product of the transmission probability per bite and the biting rate of mosquitoes and the contact rate of vector per human per unit time, while $M$ is the carrying capacity for human. Thus,

$$
\frac{d S_{h}}{d t}=\lambda_{h} N_{h}-\frac{\beta_{1} S_{h} I_{v}}{N_{v}}+c R_{h}-\frac{\lambda_{h} S_{h} N_{h}}{M}
$$

The rate of change of the population of Exposed humans is given by:

$$
\frac{d E_{h}}{d t}=\frac{\beta_{1} S_{h} I_{v}}{N_{v}}-K E_{h}-\frac{\lambda_{h} E_{h} N_{h}}{M}
$$

where $K$ is the per capita rate of progression of humans from the exposed class to the infectious class. The population of infected humans is increased by the progression of human from the exposed state to the infectious state(at a per capita rate $K$ ) and decreased by human spontaneous recovery (at a rate $\gamma$ ). It is 
reduced by the disease induced death rate(at per capita rate $\rho$ ). The population of Infected humans is given by

$$
\frac{d I_{h}}{d t}=K E_{h}-\gamma I_{h}-\rho I_{h}-\frac{\lambda_{h} I_{h} N_{h}}{M}
$$

where $\gamma$ is the per capita rate of recovery of the hosts.

The population of recovered human is generated following a human spontaneous recovery (at a rate $\gamma$ ) and decreased by loss of immunity(at a rate $c$ ). The population of recovered humans is given by

$$
\frac{d R_{h}}{d t}=\gamma I_{h}-c R_{h}-\frac{\lambda_{h} R_{h} N_{h}}{M}
$$

The population of susceptible vector is generated by birth (recruitment) of humans (at a per capita rate $\lambda_{v}$, ). It is reduced by Infection, following number of bites of a Susceptible vector on Infected human per unit time $\left(\frac{\beta_{2} S_{v} I_{h}}{N_{h}}\right)$ and also reduced by natural death at (a rate $d_{v}$ ).

$$
\frac{d S_{v}}{d t}=\lambda_{v}-\frac{\beta_{2} S_{v} I_{h}}{N_{h}}-d_{v} S_{v}
$$

The rate of change of the population of the exposed vectors is given by:

$$
\frac{d E_{v}}{d t}=\frac{\beta_{2} S_{v} I_{h}}{N_{h}}-\left(u+d_{v}\right) E_{v}
$$

where $u$ is the per capita rate of the progression of mosquitoes from the exposed state to the infectious state. The population of infected vectors is increased by the progression of vectors from the exposed state to the infectious(at a per capita rate $u$ ) and decreased by the natural death rate(at a rate $d_{v}$ ). Hence, the population of Infected vector is given by

$$
\frac{d I_{v}}{d t}=u E_{v}-d_{v} I_{v}
$$

The above leads to the following systems of equations:

$$
\begin{aligned}
\frac{d S_{h}}{d t} & =\lambda_{h} N_{h}-\frac{\beta_{1} S_{h} I_{v}}{N_{v}}+c R_{h}-\frac{\lambda_{h} S_{h} N_{h}}{M} \\
\frac{d E_{h}}{d t} & =\frac{\beta_{1} S_{h} I_{v}}{N_{v}}-K E_{h}-\frac{\lambda_{h} E_{h} N_{h}}{M} \\
\frac{d I_{h}}{d t} & =K E_{h}-\gamma I_{h}-\rho I_{h}-\frac{\lambda_{h} I_{h} N_{h}}{M} \\
\frac{d R_{h}}{d t} & =\gamma I_{h}-c R_{h}-\frac{\lambda_{h} R_{h} N_{h}}{M} \\
\frac{d S_{v}}{d t} & =\lambda_{v}-\frac{\beta_{2} S_{v} I_{h}}{N_{h}}-d_{v} S_{v} \\
\frac{d E_{v}}{d t} & =\frac{\beta_{2} S_{v} I_{h}}{N_{h}}-\left(u+d_{v}\right) E_{v} \\
\frac{d I_{v}}{d t} & =u E_{v}-d_{v} I_{v} .
\end{aligned}
$$




\subsection{The Model Equation}

$$
\begin{aligned}
\frac{d S_{h}}{d t} & =\lambda_{h} N_{h}-\frac{\beta_{1} S_{h} I_{v}}{N_{v}}+c R_{h}-\frac{\lambda_{h} S_{h} N_{h}}{M} \\
\frac{d E_{h}}{d t} & =\frac{\beta_{1} S_{h} I_{v}}{N_{v}}-K E_{h}-\frac{\lambda_{h} E_{h} N_{h}}{M} \\
\frac{d I_{h}}{d t} & =K E_{h}-\gamma I_{h}-\rho I_{h}-\frac{\lambda_{h} I_{h} N_{h}}{M} \\
\frac{d R_{h}}{d t} & =\gamma I_{h}-c R_{h}-\frac{\lambda_{h} R_{h} N_{h}}{M} \\
\frac{d S_{v}}{d t} & =\lambda_{v}-\frac{\beta_{2} S_{v} I_{h}}{N_{h}}-d_{v} S_{v} \\
\frac{d E_{v}}{d t} & =\frac{\beta_{2} S_{v} I_{h}}{N_{h}}-\left(u+d_{v}\right) E_{v} \\
\frac{d I_{v}}{d t} & =u E_{v}-d_{v} I_{v} .
\end{aligned}
$$

Subject to the boundary conditions

$$
\begin{gathered}
S_{h}(0)=S_{h, 0}, \quad E_{h}(0)=E_{h, 0}, \quad I_{h}(0)=I_{h, 0}, \\
R_{h}(0)=R_{h, 0}, \quad S_{v}(0)=S_{v, 0}, \quad E_{v}(0)=E_{v, 0}, \quad I_{v}(0)=I_{v, 0} .
\end{gathered}
$$

Defined variables and parameters in Table 2.1 and Table 2.2.

$$
\begin{aligned}
S_{h}(t) & : \text { Susceptible Human per unit time } \\
E_{h}(t) & : \text { Exposed Humans per unit time } \\
I_{h}(t) & : \text { Infected Humans per unit time } \\
R_{h}(t) & : \text { Recovered Humans per unit time } \\
S_{v}(t) & : \text { Susceptible Vector per unit time } \\
E_{v}(t) & : \text { Infected Vector per unit time } \\
I_{v}(t) & : \text { Infected Vector per unit time } \\
N_{h}(t) & : \text { Total human population per unit time } \\
N_{v}(t) & : \text { Total vector population per unit time }
\end{aligned}
$$

Table 2.2 Parameter definition for the model

$\lambda_{h}$ : The per capita extrinsic growth rate of humans

$\lambda_{v}:$ The per capita birth rate of vector

$\beta_{1}$ : The number of bites of susceptible vector on infected human per unit

$\beta_{2}$ : The number of bites on human per vector per unit time

$\gamma$ : The per capita rate of recoveries of the hosts

$u$ : per capita rate of progression of vectors from the exposed class to the infectious class 
$\rho$ : the disease induced death rate

$c$ : rate of loss of immunity by human

$K$ : per capita rate of progression of humans from the exposed class to the infectious class

$d_{v}:$ natural death rate per vector

$M$ : The carrying capacity for human.

\subsection{Basic Properties of the Model}

\subsubsection{Positivity and Boundedness of Solution}

The $S_{h} E_{h} I_{h} R_{h} S_{h}+S_{v} E_{v} I_{v}$ malaria model will be analyzed in a biologically feasible region as follows. This region should be feasible for both humans and mosquitoes populations. Hence, we have

Theorem 1. If $S_{h}(0), E_{h}(0), I_{h}(0), R_{h}(0), S_{v}(0), E_{v}(0), I_{v}(0)$ are non-negative, then so are $S_{h}(t), E_{h}(t), I_{h}(t), R_{h}(t), S_{v}(t), E_{v}(t), I_{v}(t)$ for all $t>0$. Hence

$$
\lim _{t \rightarrow \infty} \sup N_{h}(t) \leq M, \quad \lim _{t \rightarrow \infty} \sup N_{v}(t) \leq \frac{\lambda_{v}}{d v} .
$$

Moreover

$$
N_{h}(t)=S_{h}(t)+E_{h}(t)+I_{n}(t)+R_{n}(t) \text { and } N_{v}(t)=S_{v}(t)+E_{v}(t)+I_{v}(t) .
$$

Proof. Let

$$
\begin{aligned}
T_{1}=\sup \left\{t>0: S_{h}(t)>0, E_{h}(t)>0, I_{h}(t)>0\right. & , R_{h}(t)>0, \\
& \left.S_{v}(t)>0, E_{v}(t)>0, I_{v}>0\right\} .
\end{aligned}
$$

Since $S_{h}(0)>0, E_{h}>0, I_{h}(0)>0, R_{h}(0), S_{v}(0)>0, E_{v}(0)>0, I_{v}(0)>0$, then $T_{1}>(0)$. If $T_{1}<\infty$, then $S_{h}, E_{h}, I_{h}, R_{h}, S_{v}, E_{v}, I_{v}$ are equal to zero at $T_{1}$. It follows from the first equation of the system (2), that

$$
\frac{d S_{h}}{d t}=\lambda_{h} N_{h}-\frac{\beta_{1} S_{h} I_{v}}{N_{v}}+c R_{h}-\frac{\lambda_{h} S_{h} N_{h}}{M} .
$$

Thus,

$$
\frac{d S_{h}}{d t}\left\{S_{h}(t) \exp \left[\left(\frac{\lambda_{h} 0_{h} N_{h}}{M}+\frac{\beta_{1} 0_{h} I_{v}}{N_{v}}\right) t\right]=\left(c R_{h}+\lambda_{h} N_{h}\right) \times \exp \left[\left(\frac{\lambda_{h} N_{h}}{M}+\frac{\beta_{1} I_{v}}{N_{v}}\right) t\right] .\right.
$$


Hence,

$$
S_{h}\left(T_{1}\right) e^{\left[\left(\frac{\lambda_{h} N_{h}}{M}+\frac{\beta_{1} I v}{N_{v}}\right) t\right]}-S_{h}(0)=\int_{0}^{T_{1}}\left(C R_{h}+\lambda_{h} N_{h}\right) e^{\left[\left(\frac{\lambda_{h} N_{h}}{M}+\frac{\beta_{1} I_{v}}{N_{v}}\right) t\right]} d v
$$

so that

$$
\begin{aligned}
& S_{h}\left(T_{1}\right)=S_{h}(0) e^{\left[\left(\frac{\lambda_{h} N_{h}}{M}+\frac{\beta_{1} I_{v}}{N_{v}}\right) t\right]} \\
& +e^{\left[\left(\frac{\lambda_{h} N_{h}}{M}+\frac{\beta_{1} I v}{N_{v}}\right) t\right]} \times \int_{0}^{T_{1}}\left(c R_{h}+\lambda_{h} N_{h}\right) e^{\left[\left(\frac{\lambda_{h} N_{h}}{M}+\frac{\beta_{1} I v}{N_{v}}\right) t\right]} d v>0
\end{aligned}
$$

and

$$
S_{v}\left(T_{1}\right)=S_{v}(0) e^{\left[\left(\frac{\beta_{2} I_{h}}{N_{h}}+d_{v}\right) t\right]}+e^{\left[\left(\frac{\beta_{2} I_{h}}{N_{h}}+d_{v}\right) t\right]} \times \int_{0}^{T_{1}} \lambda_{v} e^{\left[\left(\frac{\beta_{2} I_{h}}{N_{h}}+d_{v}\right) v\right]} d v>0
$$

These can be similarly shown for $E_{h}>0, I_{h}>0, R_{h}>0, E_{v}>0$ and $I_{v}>0$ for all $t>0$. For the remaining part of the proof, it should be noted that $0 \leq I_{h} \leq N_{h}(t)$ and $0 \leq I_{v} \leq N_{v}(t)$.

If we add the first four equations and the last three equations of the model (1) we derive

$$
\begin{aligned}
\frac{d N_{h}(t)}{d t} & =\lambda_{h} N_{h}\left(1-\frac{N_{h}}{M}\right)-\rho I_{h} \\
\frac{d N_{v}(t)}{d t} & =\lambda_{v}-d_{v} N_{v}
\end{aligned}
$$

Therefore

$$
\begin{gathered}
\lambda_{h} N_{h}\left(1-\frac{N_{h}(t)}{M}\right)-\rho N_{h}(t) \leq \frac{d N_{h}(t)}{d t} \leq \lambda_{h} N_{h}(t)\left(1-\frac{N_{h}(t)}{M}\right) \\
\lambda_{v}-d_{v} N_{v} \leq \frac{d N_{v}(t)}{d t} \leq \lambda_{v}-d_{v} N_{v}(t) .
\end{gathered}
$$

Then,

$$
M-\frac{\rho}{\lambda_{h}} \leq \lim _{t \rightarrow \infty} \inf N_{h}(t) \leq \lim _{t \rightarrow \infty} \sup N_{v}(t) \leq M
$$

and

$$
\frac{\lambda_{v}}{d_{v}} \leq \lim _{t \rightarrow \infty} \inf N_{v}(t) \leq \lim _{t \rightarrow \infty} \sup N_{v}(t) \leq \frac{\lambda_{v}}{d_{v}} .
$$

Hence, the result. 


\subsubsection{Invariant Region}

The model equation (1) will be analyzed into biological feasible region as follows. We split the system equation (1) into two parts such as the human population: $N_{h}=S_{h}+E_{h}+I_{h}+R_{h}$ and mosquito(vector) population: $N_{v}=S_{v}+E_{v}+I_{v}$. We consider the feasible region:

$$
\begin{gathered}
\Omega=\Omega_{h} \cup \Omega_{v} \subset \mathbb{R}_{+}^{4} \times \mathbb{R}_{+}^{3} \\
\Omega_{h}=\left\{\left(S_{h}, E_{h}, I_{h}, R_{h}\right) \in \mathbb{R}_{+}^{4}: S_{h}+E_{h}+I_{h}+R_{h} \leq M\right\}
\end{gathered}
$$

and

$$
\Omega_{v}=\left\{\left(S_{v}, E_{v}, I_{v}\right) \in \mathbb{R}_{+}^{3}: S_{v}+E_{v}+I_{v} \leq \frac{\lambda_{v}}{d_{v}}\right\} .
$$

We follow this particular steps as shown below to establish the positive invariance of $\Omega$ (i.e: the solutions in $\Omega$ remain in $\Omega$ for all $t>0$ ). We give the rate of change of the human and mosquito population as follows:

$$
\begin{gathered}
\frac{d N_{h}(t)}{d t} \leq \lambda_{h} N_{h}(t)\left(1-\frac{N_{h}(t)}{M}\right) \\
\frac{d N_{v}(t)}{d t} \leq \lambda_{v}-d_{v} N_{v}(t) .
\end{gathered}
$$

By the standard comparison theorem we showed that

$$
N_{h}(t) \leq \frac{M N_{h}(0)}{N_{h}(0)+\left(M-N_{h}(0)\right) e^{-t \lambda}}
$$

and

$$
N_{v}(t) \leq N_{v}(0) e^{-d_{v} t}+\frac{b_{v}}{d_{v}}\left(1-e^{-d_{v} t}\right) .
$$

In particular,

$$
N_{h}(t) \leq \frac{M(0)}{1+(M-1) e^{-t \lambda_{h}}} \text { and } N_{v}(t) \leq \frac{\lambda_{v}}{d_{v}}
$$

whenever $N_{h}(0) \leq 1$ and $N_{v}(0) \leq 1$ respectively.

Therefore, the region $\Omega$ is positively invariant. Wherefore, it is sufficient to consider the dynamics of the flow generated by (1) in $\Omega$. In this region, the model can be considered to be epidemiologically and mathematically well posed. Hence, every solution of the model (1) with initial conditions in $\Omega$ remains in $\Omega$ for all $t>0$. 


\section{The Disease-Free Equilibrium and its Stability}

In this section 3, we study the stability of the disease-free equilibrium $P_{0}$. Our model equation (1) has a Disease-Free Equilibrium given by

$$
P_{0}=\left(S_{h}^{*}, E_{h}^{*}, I_{h}^{*}, R_{h}^{*}, S_{v}^{*}, E_{v}^{*}, I_{v}^{*}\right)=\left(M, 0,0,0, \frac{b_{v}}{d v}, 0,0\right) .
$$

We try to explore the local stability of $P_{0}$ first using the next generation matrix (operator method). Using the notation $F$ as the non-negative matrix of new infection terms and the $M$-matrix, $V$, of transition terms associated with our model (1) are given respectively by

$$
F=\left(\begin{array}{cccc}
0 & 0 & 0 & \frac{\beta_{1} M}{N_{h}} \\
0 & 0 & 0 & 0 \\
0 & \frac{\beta_{2} b_{v}}{d_{v} N_{v}} & 0 & 0 \\
0 & 0 & 0 & 0
\end{array}\right)
$$

and

$$
V=\left(\begin{array}{cccc}
K & 0 & 0 & 0 \\
-K & \gamma+\rho & 0 & 0 \\
0 & 0 & u+d_{v} & 0 \\
0 & 0 & -u & d_{v}
\end{array}\right)
$$

It follows that the basic reproduction number of the model equation (1), denoted by $R_{0}$ is given by (where $\rho$ denotes the spectral radius)

$$
\begin{gathered}
R_{0}=\rho\left(F V^{-1}\right) \sqrt{\frac{\beta_{2} \beta_{1} \lambda_{v} M u}{N_{v} N_{h}(\gamma u+\rho)\left(\rho+\gamma^{2} u+\gamma d_{v}\right) d_{v}^{2}}} . \\
R_{0}^{2}=\frac{\beta_{2} \beta_{1} \lambda_{v} M u}{N_{v} N_{h}(\gamma+\rho)\left(u+d_{v}\right) d_{v}^{2}} .
\end{gathered}
$$

We using the theorem below (Theorem 1) we further established our result.

Theorem 2. The DFE of the model equation (1), given by $P_{0}$, is locally asymptotically stable (LAS) if $R_{0}<1$, and unstable if $R_{0}>1$.

This implies that malaria can be eliminated from the community when $R_{0}<1$ epidemologically. If $R_{0}<1$ then, average of an infected individual produce less than one new infected individual over the period of its infectious 
period and the infection dies out. For $R_{0}$ to be less than one, this will only be possible if $\beta_{1}$ and $\beta_{2}$ (rate of transmission) are decreasing without limits. But if $R_{0}>1$, then each infected individual produce an average of more than one infection and the disease persist and invade the population.

Let

$$
\begin{gathered}
J_{P_{0}}= \\
\left(\begin{array}{ccccccc}
-\lambda_{h} N_{h} & 0 & 0 & c & 0 & 0 & \frac{\beta_{1} M}{N_{v}} \\
0 & -k-\frac{\lambda_{h} N_{h}}{M} & 0 & 0 & 0 & 0 & \frac{\beta_{1} M}{N_{v}} \\
0 & k & -\gamma-\rho-\frac{\lambda_{h} N_{h}}{M} & 0 & 0 & 0 & 0 \\
0 & 0 & \gamma & -c-\frac{\lambda_{h} N_{h}}{M} & 0 & 0 & 0 \\
0 & 0 & -\frac{\beta_{2} \lambda_{v}}{d_{v} N_{h}} & 0 & -d_{v} & 0 & 0 \\
0 & 0 & \frac{\beta_{2} \lambda_{v}}{d_{v} M_{h}} & 0 & 0 & -u-d_{v} & 0 \\
0 & 0 & 0 & 0 & 0 & u & -d_{v}
\end{array}\right) .
\end{gathered}
$$

We derive $\left|J_{P_{0}}-\lambda I\right|=0$.

The characteristic polynomial to $J_{P_{0}}$ is,

$$
\begin{aligned}
F(\lambda) & =\lambda^{7}+a_{6} \lambda^{6}+a_{5} \lambda^{5}+a_{4} \lambda^{4}+a_{3} \lambda^{3}+a_{2} \lambda^{2}+a_{1} \lambda+a_{0} \\
& =0,
\end{aligned}
$$

where

$$
\begin{aligned}
a_{6}=\frac{\left(3 d_{v}+u+c+\gamma+\rho+k+\lambda_{h} N_{h}\right) M+3 \lambda_{h} N_{h}}{M} \\
a_{5}=\frac{1}{M^{2}}\left(N_{h}\left(c+3 d_{v}+u+\rho+k+\gamma\right) \lambda_{h}+3 d_{v}^{2}+(3 \rho+3 k+2 u+3 \gamma+3 c) d_{v}\right. \\
+(\gamma+\rho+u+k) c+(\gamma+\rho+u) k+u(\gamma+\rho) M^{2} \\
\left.+2 N_{h} \lambda_{h}\left(\frac{3}{2} \lambda_{h} N_{h}+k+c+\frac{9}{2} d_{v}+\frac{3}{2} u+\rho+\gamma\right) M+3 \lambda_{h}^{2} N_{h}^{2}\right) \\
\\
a_{4}=\frac{1}{M^{3}}\left(\left(3 d_{v}^{2}+(2 u+3 c+3 \gamma+3 \rho+3 k) d_{v}+(\gamma+\rho+k+u) c+(\rho+\gamma+u) k\right.\right. \\
\left.+u(\gamma+\rho)) N_{h} \lambda_{h}+d_{v}^{3}+(3 c+u+3 \gamma+3 k+3 \rho)\right) d_{v}^{2} \\
+((2 u+3 \gamma+3 \rho+3 k) c+(2 u+3 \gamma+3 \rho) k+2 u(\gamma+\rho)) d_{v} \\
+((\rho+\gamma+u) k+u(\gamma+\rho)) c+u k(\gamma+\rho)) M^{3} \\
+\left(2\left(\rho+\frac{3}{2} u+\gamma+\frac{9}{2} d_{v}+k+c\right) N_{h} \lambda_{h}+9 d_{v}^{2}+(6 u+6 \rho+6 \gamma+6 c+6 k) d_{v}\right. \\
+(\rho+\gamma+2 u+k) c+(\gamma+2 u+\rho) k+2 u(\gamma+\rho) \lambda_{h} N_{h} M^{2}
\end{aligned}
$$




$$
\begin{aligned}
& \left.+\lambda_{h}^{2} M_{h}^{2}\left(3 \lambda_{h} N_{h}+3 u+9 d_{v}+k+\gamma+\rho+c\right) M+\lambda_{h}^{3} N_{h}^{3}\right), \\
& a_{3}=\frac{1}{d_{v}\left(N_{h} N_{v} M^{3}\right)}\left(d_{v} N_{h}^{5} N_{v} \lambda_{h}^{4}+\left((9 M+3) d_{v}\right.\right. \\
& +(\gamma+\rho+c+k+3 u) M+u) \lambda_{h}^{3} N_{v} d N \\
& +\lambda_{h}^{2} N_{v} M\left((9+9 M) d_{v}^{2}\right. \\
& +((6 \rho+6 \gamma+6 c+6 k+6 u) M+3 \gamma+3 \rho+3 c+6 u+3 k) d_{v} \\
& +((2 \rho+2 k+2 c+2 \gamma) u+(\gamma+\rho+c) k+c(\gamma+\rho)) M \\
& +u(\gamma+\rho+c+k)) d_{v} N_{h}^{3} \\
& +\lambda_{h} N_{v} M^{2} d_{v}\left((M+3) d_{v}^{3}\right. \\
& +((3 k+3 c+3 \gamma+u+3 \rho) M+6 \gamma+3 u+6 \rho+6 c+6 k) d_{v}^{2} \\
& +(3 \gamma+3 \rho+3 c) k+3 c(\gamma+\rho)) M+(4 k+4 c+4 \rho+4 \gamma) u \\
& +(3 \gamma+3 \rho+3 c) k \\
& +3 c(\gamma+\rho)) d_{v}+(((\gamma+\rho+c) k+c(\gamma+\rho)) u+c k(\gamma+\rho)) M \\
& +((\gamma+\rho+c) k+c(\gamma+\rho)) u) N_{h}^{2}+\left((\gamma+\rho+c+k) d_{v}^{3}\right. \\
& (u(\gamma+\rho+c+k)+(3 \gamma+3 \rho+3 c) k+3 c(\gamma+\rho)) d_{v}^{2}+(((2 \rho+2 c+2 \gamma) k \\
& +c(\gamma+\rho)) u+3 c k(\gamma+\rho)) N_{v} M^{3} d_{v} N_{h}-u \beta_{2} \lambda_{v} k \beta_{1} M^{4} \text {, } \\
& a_{2}=\frac{1}{d_{v} N_{h} N_{v} M^{3}}\left(d_{v} N_{v} \lambda_{h}^{4}\left(3 d_{v}+u\right) N_{h}^{5}\right. \\
& -\left((9 M+3) d_{v}^{2}+((3 c+3 \rho+3 k+3 \gamma+6 u) M+2 u) d_{v}\right. \\
& +M u(\gamma+\rho+c+k) d_{v} \lambda_{h}^{3} N_{v} N_{h}^{4}-M d_{v} \lambda_{h}^{2}\left((3+3 M) d_{v}^{3}\right. \\
& +((6 c+6 \rho+6 \gamma+6 k+3 u) M+3 u+3 c+3 \rho+3 k+3 \gamma) d_{v}^{2} \\
& +(((4 k+4 c+4 \rho+4 \gamma) u \\
& +(3 \gamma+3 \rho+3 c) k+3 c(\gamma+\rho)) M+2 u(\gamma+\rho+c+k)) d_{v} \\
& +M u((\gamma+\rho+c) k \\
& +c(\gamma+\rho))) N_{v} N_{h}^{3}+\left((\gamma+\rho+c+k)(2+M) d_{v}^{3}+((u(\gamma+\rho+c+k)\right. \\
& +(3 \gamma+3 \rho+3 c) k+3 c(\gamma+\rho)) M+(2 \rho+2 c+2 \gamma) u \\
& +(3 \gamma+3 \rho+3 c) k+3 c(\gamma+\rho)) d_{v}^{2} \\
& +((((2 \rho+2 c+2 \gamma) k+2 c(\gamma+\rho)) u+3 c k(\gamma+\rho) M+2((\gamma+\rho+c) k \\
& +c(r+\rho)) u) d_{v} \\
& +\operatorname{Muck}(\gamma+\rho)) M^{2} d_{v} \lambda_{h} N_{v} N_{h}^{2}+M^{3}\left(((\gamma+\rho+c) k+c(\gamma+\rho)) N_{v} d_{v}^{4}\right.
\end{aligned}
$$




$$
\begin{aligned}
& +(((\gamma+\rho+c) k+c(\gamma+\rho)) u+3 c k(\gamma+\rho)) N_{v} d_{v}^{3}+2 u c k N_{v}(\gamma+\rho) d_{v}^{2} \\
& \left.\left.\left.+k \beta_{2} \lambda_{v} u \beta_{1} \lambda_{h}(1+M)\right) N_{h}+M^{4} k \beta_{2} \lambda_{v} u \beta_{1}\left(d_{v}+c\right)\right)\right) \text {, } \\
& a_{1}=\frac{1}{d_{v} N_{h} N_{v} M^{3}}\left(2 N_{v}\left(\frac{3}{2} d_{v}+u\right) \lambda_{h}^{4} d_{v}^{2} N_{h}^{5}+2\left(\left(\frac{3}{2} M+\frac{1}{2} d_{v}^{2}\right.\right.\right. \\
& \left.+\left(\left(\frac{3}{2} \rho+\frac{3}{2} k+\frac{3}{2} u+\frac{3}{2} \gamma+\frac{3}{2}\right) M+\frac{1}{2} u\right) d_{v}+M_{u}(\gamma+\rho+c+k)\right) N_{v} \lambda_{h}^{3} d_{v}^{2} N_{h}^{4} \\
& +2 M N_{v} \lambda_{h}^{2}\left((\gamma+\rho+c+k)\left(M+\frac{1}{2}\right) d_{v}^{2}+((u(\gamma+\rho+c+k)\right. \\
& \left.+\left(\frac{3}{2} \rho+\frac{3}{2} \gamma+\frac{3}{2} c\right) k+\frac{3}{2} c(\gamma+\rho)\right) M+\frac{1}{2}(u(\gamma+\rho+c+k)) d_{v} \\
& +M u((\gamma+\rho+c) k+c(\gamma+\rho))) d_{v}^{2} N_{h}^{3}+2\left(\frac{1}{2}((\gamma+\rho+c) k\right. \\
& +c(\gamma+\rho)) N_{v}(1+M) d_{v}^{4} \\
& +\frac{1}{2} N_{v}((((\gamma+\rho+c) k+c(\gamma+\rho)) u+3 c k(\gamma+\rho)) M+((\gamma+\rho+c) k \\
& +c(\gamma+\rho)) u) d_{v}^{3} \\
& \left.+M u c K N_{v}(\gamma+\rho) d_{v}^{2}-\frac{1}{2} M u \beta_{2} \lambda_{v} K \beta_{1} \lambda_{h}\right) M^{2} \lambda_{h} N_{h}^{2} \\
& -M^{3} K\left(c N_{v}(\gamma+\rho) d_{v}^{4}\right. \\
& +u\left(N_{v}(\gamma+\rho) d_{v}^{3}-u \beta_{2} \lambda_{v} \beta_{1} \lambda_{h}(1+M) d_{v}\right. \\
& \left.\left.-M \beta_{2} \lambda_{v} u \beta_{1} c \lambda_{h}\right) N_{h}-k \beta_{2} \lambda_{2} u \beta_{1} M^{4} d_{v} c\right) \text {. } \\
& a_{0}=\left(\left(d_{v}^{2} N_{v} \lambda_{h}^{2}\left(d_{v}+u\right) N_{h}^{3}+M d_{v}^{2} N_{v} N_{h}\left(d_{v}+u\right)(\rho+\gamma+k) N_{h}^{2}\right.\right. \\
& \left.\left.+M^{2} k d_{v}^{2} N_{v}(\gamma+\rho)\left(d_{v}+u\right) N_{h}-k \beta_{2} \lambda_{v} u \beta_{1} M^{3}\right)\left(C M+\lambda_{h} N_{h}\right)\right)\left(N_{v} M^{3}\right)^{-1} \text {, } \\
& a_{0}=\left(d_{v}^{2} N_{v} \lambda_{h} d_{v} N_{h}^{3}+d_{v}^{2} N_{v} \lambda_{h}^{2} u N_{h}^{3}+M d_{v}^{2} N_{v} N_{h}\left(d_{v}+u\right)(\rho+\gamma+k) N_{h}^{2}\right. \\
& \left.+M^{3} k \beta_{2} \beta_{1} \lambda_{v} u\left[\frac{1}{R_{0}^{2}}-1\right]\right)\left(c M+\lambda_{h} N_{h}\right) \text {. }
\end{aligned}
$$

If $R_{0}<1$, we clearly have that $a_{0}>0$. Thus, according to the Routh Hurwitz condition $P_{0}$ is locally asymptotically stable when $a_{0}>0$. From $a_{0}>0$ it implies that

$$
\begin{aligned}
\frac{\left(M+\lambda_{h} N_{h}\right)}{N_{v} M^{3}}\left[d_{v}^{3} N_{v} \lambda_{h} N_{h}^{3}\right. & +d_{v}^{2} N_{v} \lambda_{h}^{2} u N_{h}^{3}+M d_{v}^{2} N_{v} N_{h}\left(d_{v}+u\right)(\rho+\gamma+k) N_{h}^{2} \\
& \left.+M^{3} k \beta_{2} \beta_{1} \lambda_{v} u\left[\frac{1}{R_{0}^{2}}-1\right]\right]>0 .
\end{aligned}
$$


Therefore $R_{0}<1$.

Hence the disease free equilibrium of the Malaria Model (1) is locally Asymptotically stable.

Global Asymptotic Stability for the Disease-Free Equilibrium Point

Theorem 3. The disease-free equilibrium, $P_{0}$ is globally asymptotically stable if $R_{m} \leq 1$.

The Disease-Free Equilibrium (DFE) exist only if given that $R_{m} \leq 1$.

Proof. We consider the Lyapunov function

$$
\begin{aligned}
L(t)= & \frac{1}{2}\left(S_{n}-\bar{S}_{n}\right)^{2}+\frac{1}{2}\left(E_{h}-\bar{E}_{n}\right)^{2}+\frac{1}{2}\left(I_{n}-\bar{I}_{n}\right)^{2}+\frac{1}{2}\left(R_{n}-\bar{R}_{n}\right)^{2} \\
& +\frac{1}{2}\left(S_{v}-\bar{S}_{v}\right)^{2}+\frac{1}{2}\left(E_{v}-\bar{E}_{v}\right)^{2}+\frac{1}{2}\left(I_{v}-\bar{I}_{v}\right)^{2} .
\end{aligned}
$$

We differentiate the Lyapunov function, $L$, which gives

$$
\begin{aligned}
\frac{d L}{d t}= & \left(S_{h}-\bar{S}_{h}\right) \frac{d S_{h}}{d t}+\left(E_{h}-\bar{E}_{h}\right) \frac{d E_{h}}{d t}+\left(I_{h}-\bar{I}_{h}\right) \frac{d E_{h}}{d t}+\left(R_{n}-\bar{R}_{h}\right) \frac{d R_{h}}{d t} \\
& +\left(S_{v}-\bar{S}_{v}\right) \frac{d S_{v}}{d t}+\left(E_{v}-\bar{E}_{v}\right) \frac{d E_{v}}{d t}+\left(I_{v}-\bar{I}_{v}\right) \frac{d I_{v}}{d t} \\
= & \left(S_{h}-\bar{S}_{h}\right)\left[\lambda_{h} N_{h}-\frac{\beta_{1}\left(S_{h}-\bar{S}_{h}\right)\left(I_{v}-\bar{I}_{v}\right)}{N_{v}}+c\left(R_{h}-\bar{R}_{h}\right)-\frac{\lambda_{h}\left(S_{h}-\bar{S}_{h}\right) N_{h}}{M}\right] \\
& +\left(E_{h}-\bar{E}_{h}\right)\left[\frac{\beta_{1}\left(S_{h}-\bar{S}_{h}\right)\left(I_{v}-\bar{I}_{v}\right)}{N_{v}}-k\left(E_{h}-\bar{E}_{h}\right)-\frac{\lambda_{h}\left(E_{h}-\bar{E}_{h}\right) N_{h}}{M}\right] \\
& +\left(I_{h}-\bar{I}_{h}\right)\left[I c\left(E_{h}-\bar{E}_{h}\right)-\gamma\left(I_{h}-\bar{I}_{h}\right)-\rho\left(I-\bar{I}_{h}\right)-\frac{\lambda_{h}\left(I_{h}-\bar{I}_{h}\right) N_{h}}{M}\right] \\
& +\left(R_{h}-\bar{R}_{h}\right)\left[\gamma\left(I_{h}-\bar{I}_{h}\right)-c\left(R_{h}-\bar{R}_{h}\right)-\frac{\lambda_{h}\left(R_{h}-\bar{R}_{h}\right) N_{h}}{M}\right] \\
& +\left(S_{v}-\bar{S}_{v}\right)\left[\lambda_{v}-\frac{\beta_{2}\left(S_{v}-\bar{S}_{v}\right)\left(I_{h}-\bar{I}_{h}\right)}{N_{h}}-d_{v}\left(S_{v}-\bar{S}_{v}\right)\right] \\
& +\left(E_{v}-\bar{E}_{v}\right)\left[\frac{\beta_{2}\left(S_{v}-\bar{S}_{v}\right)\left(I_{h}-\bar{I}_{h}\right)}{N_{h}}-\left(u+d_{v}\right)\left(E_{v}+\bar{E}_{v}\right)\right] \\
& +\left(I_{v}-\bar{I}_{v}\right)\left[u\left(E_{v}-\bar{E}_{v}\right)-d_{v}\left(I_{v}-\bar{I}_{v}\right)\right]
\end{aligned}
$$




$$
\begin{aligned}
& d L d t=\lambda_{h} N_{h}\left(S_{h}-\bar{S}_{h}\right)+\frac{\beta_{1} \bar{S}_{h} I_{v}}{N_{v}}\left(S_{h}-\bar{S}_{h}\right)+\frac{\lambda_{h} \bar{S}_{h} N_{h}}{M}\left(S_{h}-\bar{S}_{h}\right)+c R_{h}\left(S_{h}-\bar{S}_{h}\right) \\
& +k E_{h}\left(E_{h}-\bar{E}_{h}\right)+\frac{\lambda_{h} E_{h} N_{h}}{M}\left(E_{h}-\bar{E}_{h}\right)+\gamma I_{h}\left(I_{h}-\bar{I}_{h}\right)+\rho \bar{I}_{h}\left(I_{h}-\bar{I}_{h}\right) \\
& +\frac{\lambda_{h} \bar{I}_{h} N_{h}}{M}\left(I_{h}-\bar{I}_{h}\right)+c \bar{R}_{h}\left(R_{h}-\bar{R}_{h}\right)+\frac{\lambda_{h} \bar{R}_{h} N_{h}}{M}\left(R_{h}-\bar{R}_{h}\right)+\lambda_{v}\left(S_{v}-\bar{S}_{v}\right) \\
& +\frac{\beta_{2} S_{v} \bar{I}_{h}}{N_{h}}\left(S_{v}-\bar{S}_{v}\right)+\frac{\beta_{2} \bar{S}_{v} I_{h}}{N_{h}}\left(S_{v}-S_{v}\right)+d_{v} \bar{S}_{v}\left(S_{v}-\bar{S}_{v}\right)+u \bar{E}_{v}\left(E_{v}-\bar{E}_{v}\right) \\
& +d_{v} \bar{E}_{v}\left(E_{v}-\bar{E}_{v}\right)+d_{v} \bar{I}_{v}\left(I_{v}-\bar{I}_{v}\right)+\frac{\beta_{1} S_{h} \bar{I}_{v}}{N_{v}}\left(E_{h}-\bar{E}_{h}\right)+\frac{\beta_{1} \bar{S}_{h} I_{v}}{N_{v}}\left(E_{h}-\bar{E}_{h}\right) \\
& -\frac{\beta_{1} S_{h} I_{v}}{N_{v}}\left(S_{h}-\bar{S}_{h}\right)-\frac{\beta_{1} S_{h} I_{v}}{N_{v}}\left(S_{h}-\bar{S}_{h}\right)-\frac{\beta_{1} \bar{S}_{h} I_{v}}{N_{v}}\left(S_{h}-\bar{S}_{h}\right)-c R_{h}\left(S_{h}-\bar{S}_{h}\right) \\
& -\frac{\lambda_{h} S_{h} N_{h}}{M}\left(S_{h}-\bar{S}_{h}\right)-\frac{\beta_{1} S_{h} I_{v}}{N_{v}}\left(E_{h}-\bar{E}_{h}\right)-\frac{\beta_{1} \bar{S}_{h} I_{v}}{N_{v}}\left(E_{h}-\bar{E}_{h}\right)-\frac{\beta_{1} \bar{S}_{h} I_{v}}{N_{v}}\left(E_{h}-\bar{E}_{h}\right) \\
& -k E_{h}\left(E_{h}-\bar{E}_{h}\right)-\frac{\lambda_{h} E_{h} N_{h}^{v}}{M}\left(E_{h}-\bar{E}_{h}\right)-k E_{h}\left(I_{h}-\bar{I}_{h}\right)-\gamma I_{h}\left(I_{h}-\bar{I}_{h}\right)-\rho I_{h}\left(I_{h}-\bar{I}_{h}\right) \\
& -\frac{\lambda_{h} I_{h} N_{h}}{M}\left(I_{h}-\bar{I}_{h}\right)-\gamma \bar{I}_{h}\left(R_{h}-\bar{R}_{h}\right)-c R_{h}\left(R_{h}-\bar{R}_{h}\right)-\frac{\lambda R_{h} N_{h}}{M}\left(R_{h}-\bar{R}_{h}\right) \\
& -\frac{\beta_{2} S_{v} I_{h}}{N_{h}}\left(S_{v}-\bar{S}_{v}\right)-\frac{-\beta_{2} \bar{S}_{v} I_{h}}{N_{v}}\left(S_{v}-\bar{S}_{v}\right)-\frac{\beta_{2} \bar{S}_{v} I_{h}}{N_{h}}\left(S_{v}-\bar{S}_{v}\right)-d_{v} S_{v}\left(S_{v}-\bar{S}_{v}\right) \\
& -\frac{\beta_{2} \bar{S}_{v} I_{h}}{N_{v}}\left(E_{v}-\bar{E}_{v}\right)-\frac{\beta_{2} S_{v} I_{h}}{N_{h}}\left(E_{v}-\bar{E}_{v}\right)-\frac{\beta_{2} \bar{S}_{v} I_{h}}{N_{h}}\left(E_{v}-\bar{E}_{v}\right)-u E_{v}\left(E_{v}-\bar{E}_{v}\right) \\
& -d_{v} E_{v}\left(E_{v}-\bar{E}_{v}\right)-u \bar{E}_{v}\left(I_{v}-\bar{I}_{v}\right)-d_{v} I_{v}\left(I_{v}-\bar{I}_{v}^{h}\right) \text {. }
\end{aligned}
$$

The $n$ by collecting positive terms together and negative terms together from the above the equation, we get

$$
\frac{d L}{d t}=P-Q
$$

where

$$
\begin{aligned}
P= & \lambda_{h} N_{h}\left(S_{h}-\bar{S}_{h}\right)+\frac{\beta_{1} \bar{S}_{h} I_{v}}{N_{v}}\left(S_{h}-\bar{S}_{h}\right)+\frac{\lambda_{h} \bar{S}_{h} N_{h}}{M}\left(S_{h}-\bar{S}_{h}\right) \\
& +c R_{h}\left(S_{h}-\bar{S}_{h}\right)+k E_{h}\left(E_{h}-\bar{E}_{h}\right)+\frac{\lambda_{h} E_{h} N_{h}}{M}\left(E_{h}-\bar{E}_{h}\right)+\gamma I_{h}\left(I_{h}-\bar{I}_{h}\right)+\rho \bar{I}_{h}\left(I_{h}-\bar{I}_{h}\right) \\
& +\frac{\lambda_{h} \bar{I}_{h} N_{h}}{M}\left(I_{h}-\bar{I}_{h}\right)+c \bar{R}_{h}\left(R_{h}-\bar{R}_{h}\right)+\frac{\lambda_{h} \bar{R}_{h} N_{h}}{M}\left(R_{h}-\bar{R}_{h}\right)+\lambda_{v}\left(S_{v}-\bar{S}_{v}\right) \\
& +\frac{\beta_{2} S_{v} \bar{I}_{h}}{N_{h}}\left(S_{v}-\bar{S}_{v}\right)+\frac{\beta_{2} \bar{S}_{v} I_{h}}{N_{h}}\left(S_{v}-\bar{S}_{v}\right)+d_{v} \bar{S}_{v}\left(S_{v}-\bar{S}_{v}\right)+u \bar{E}_{v}\left(E_{v}-\bar{E}_{v}\right) \\
& +d_{v} \bar{E}_{v}\left(E_{v}-\bar{E}_{v}\right)+d_{v} \bar{I}_{v}\left(I_{v}-\bar{I}_{v}\right)+\frac{\beta_{1} S_{h} \bar{I}_{v}}{N_{v}}\left(E_{h}-\bar{E}_{h}\right)+\frac{\beta_{1} \bar{S}_{h} I_{v}}{N_{v}}\left(E_{h}-\bar{E}_{h}\right) .
\end{aligned}
$$




$$
\begin{aligned}
Q= & \frac{\beta_{1} S_{h} I_{v}}{N_{v}}\left(S_{h}-\bar{S}_{h}\right)+\frac{\beta_{1} S_{h} I_{v}}{N_{v}}\left(S_{h}-\bar{S}_{h}\right)+\frac{\beta_{1} \bar{S}_{h} I_{v}}{N_{v}}\left(S_{h}-\bar{S}_{h}\right)+c R_{h}\left(S_{h}-\bar{S}_{h}\right) \\
& +\frac{\lambda_{h} S_{h} N_{h}}{M}\left(S_{h}-\bar{S}_{h}\right)+\frac{\beta_{1} S_{h} I_{v}}{N_{v}}\left(E_{h}-\bar{E}_{h}\right)+\frac{\beta_{1} \bar{S}_{h} I_{v}}{N_{v}}\left(E_{h}-\bar{E}_{h}\right)+\frac{\beta_{1} \bar{S}_{h} I_{v}}{N_{v}}\left(E_{h}-\bar{E}_{h}\right) \\
& +k E_{h}\left(E_{h}-\bar{E}_{h}\right)+\frac{\lambda_{h} E_{h} N_{h}}{M}\left(E_{h}-\bar{E}_{h}\right)+k E_{h}\left(I_{h}-\bar{I}_{h}\right)+\gamma I_{h}\left(I_{h}-\bar{I}_{h}\right)-\rho I_{h}\left(I_{h}-\bar{I}_{h}\right) \\
& +\frac{\lambda_{h} I_{h} N_{h}}{M}\left(I_{h}-\bar{I}_{h}\right)+\gamma I_{h}\left(R_{h}-\bar{R}_{h}\right)+c R_{h}\left(R_{h}-\bar{R}_{h}\right)+\frac{\lambda_{h} R_{h} N_{h}}{M}\left(R_{h}-\bar{R}_{h}\right) \\
& +\frac{\beta_{2} S_{v} I_{h}}{N_{v}}\left(S_{v}-\bar{S}_{v}\right)+\frac{\beta_{2} \bar{S}_{v} I_{v}}{N_{v}}\left(S_{v}-\bar{S}_{v}\right)+\frac{\beta_{2} \bar{S}_{v} I_{h}}{N_{h}}\left(S_{h}-\bar{S}_{h}\right)+d_{v} S_{v}\left(S_{v}-\bar{S}_{v}\right) \\
& +\frac{\beta_{2} \bar{S}_{v} I_{h}}{N_{v}}\left(E_{v}-\bar{E}_{v}\right)+\frac{\beta_{2} \bar{S}_{v} I_{h}}{N_{v}}\left(E_{v}-\bar{E}_{v}\right)+\frac{\beta_{2} S_{v} I_{h}}{N_{h}}\left(E_{h}-\bar{E}_{h}\right)+u E_{v}\left(E_{v}-\bar{E}_{v}\right) \\
& +d_{v} E_{v}\left(E_{v}-\bar{E}_{v}\right)+u \bar{E}_{v}\left(I_{v}-\bar{I}_{v}\right)+d_{v} I_{v}\left(I_{v}-\bar{I}_{v}\right) .
\end{aligned}
$$

We realized that the quantities $\bar{S}_{h}, \bar{E}_{h}, \bar{I}_{h}, \bar{R}_{h}, \bar{S}_{v}, \bar{E}_{v}, \bar{I}_{v}$ are the disease-free equilibrium states.

Thus, we have shown that $i \leq 0$ provided $S_{h}>\bar{S}_{h}, E_{h}>\bar{E}_{h}, I_{h}>\bar{I}_{h}, R_{h}>$ $\bar{R}_{h}, S_{v}>\bar{S}_{v}, E_{v}>\bar{E}_{v}, I_{v}>\bar{I}_{v}$.

We find that it is important to note that, $L^{*}=0$ only at the disease-free equilibrium point $P_{0}$.

\subsection{The Endemic Equilibrium and its Stability}

In this section, we study rigorously the stability of the endemic equilibrium $P_{1}$ of Model (1).

Theorem 4. If $R_{0}>0$, then the endemic equilibrium $P_{1}$ of the model (1) is locally Asymptotic stable (LAS) in $\Omega$.

Proof. We hereby linearize the system (1) around the endemic equilibrium $P_{1}$. The matrix of the linearization method of the system (1) at the endemic equilibrium

$$
P_{1}=\left(S_{h}^{* *}, E_{h}^{* *}, I_{h}^{* *}, R_{h}^{* *}, S_{h}^{* *}, E_{v}^{* *}, I_{v}^{* *}\right)
$$

is obtained.

\subsection{Existence of the Endemic Equilibrium}

Calculating the endemic equilibrium point, we obtain

$$
\begin{aligned}
S_{h}^{*} & =\frac{\left[\left(M c+\lambda_{h} N_{h}\right)\left(M N_{v} N_{h} \lambda_{h}\right)+c M^{2} N_{v} \gamma I_{h}^{2}\right]\left(d_{v} N_{h}^{2}\left(u+d_{v}\right)\right)}{\left(M c+\lambda_{h} N_{h}\right)\left[M \beta_{1} u \beta_{2}^{2} I_{h}^{*^{2}}+M \beta_{1} \beta_{2} d_{v} N_{h} J_{h}^{*}+d_{v} N_{h}^{2}\left(u+d_{v}\right) N_{v} \lambda_{h} N_{h}\right]} \\
E_{h}^{*} & =\frac{M \beta_{1} d_{v} N_{h}^{2}\left(u+d_{v}\right)\left[M^{2} N_{v} N_{h} c \lambda_{h}+M N_{v} N_{h}^{2} \lambda_{h}^{2}+c M^{2} N_{v} \gamma I_{h}^{*}\right] I_{v}^{*}}{N_{v}\left(M_{k}+\lambda_{h} N_{h}\right)\left(M c+\lambda_{h} N_{h}\right)\left[M \beta_{1} \beta_{2}^{2}-u I_{h}^{*^{2}}+M \beta_{1} \beta_{2} d v N_{h} I_{h}^{*}+d_{v} N_{h}^{3} N_{v} \lambda_{h}\right]}
\end{aligned}
$$




$$
\begin{aligned}
I_{h}^{*} & =\frac{M K E_{h}^{*}}{M \gamma+M \rho+\lambda_{h} N_{h}} \\
R_{h}^{*} & =\frac{M \gamma I_{h}^{*}}{M c+\lambda_{h} N_{h}}
\end{aligned}
$$

$$
\begin{aligned}
S_{v}^{*} & =\frac{N_{h} \lambda_{v}}{\beta_{2} I_{h}^{*}+d_{v} N_{h}} \\
E_{v}^{*} & =\frac{\beta_{2} S_{v}^{*} I_{h}^{*}}{N_{h}\left(u+d_{v}\right)} \\
I_{v}^{*} & =\frac{u E_{v}^{*}}{d_{v}} .
\end{aligned}
$$

The epidemic equilibrium satisfies the following polynomials

$$
F\left(I_{h}^{*}\right)=A\left(I_{h}^{*}\right)^{3}+B\left(I_{h}^{*}\right)^{2}+C\left(I_{h}^{*}\right)
$$

where $I_{h}^{*}=0$ or $A\left(I_{h}^{*}\right)^{2}+B\left(I_{h}^{*}\right)+C=0$ and

$$
\begin{aligned}
A= & \left(M N_{v} \gamma+M N_{v} \rho+\lambda_{h} N_{v} N_{h}\right)\left(M^{2} K c+M K \lambda_{h} N_{h}+M c \lambda_{h} N_{h}+\lambda_{h}^{2} N_{h}^{2}\right) \\
& M \beta_{1} \beta_{2}^{2} u-M^{4} K \beta_{1} d_{v} N_{h}^{2} N_{v} \gamma u \beta_{2}^{2}\left(u+d_{v}\right), \\
B= & \left(M N_{v} \gamma+M N_{v} \rho+\lambda_{h} N_{v} N_{h}\right)\left(M^{2} K c+M K \lambda_{h} N_{h}+M c \lambda_{h} N_{h}+\lambda_{h}^{2} N_{h}^{2}\right) M \beta_{1} \beta_{2} d_{v} N_{h} \\
& -M^{3} K \beta_{1} d_{v} N_{h}^{3} u \beta_{2}^{2} N_{v} c \lambda_{h}\left(u+d_{v}\right)-M^{3} K \beta_{1} d_{v} N_{h}^{3} u \beta_{2}^{2} N_{v} \lambda_{h}^{2}\left(u+d_{v}\right) \\
& -M^{4} K \beta_{1} \beta_{2}^{2} d_{v} N_{h}^{4} N_{v} c \lambda_{h}^{2} u\left(u+d_{v}\right)-M^{4} K \beta_{1} \beta_{2} N_{h}^{3} d_{v}^{2} \gamma N_{v} c\left(u+d_{v}\right), \\
C= & \left(M N_{v} \gamma+M N_{v} \rho+\lambda_{h} N_{v} N_{h}\right)\left(M^{2} k c+M k \lambda_{h} N_{h}+M c \lambda_{h} N_{h}+\lambda_{h}^{2} N_{h}^{2}\right)\left(d_{v} N_{h}^{2} N_{v} \lambda_{h}\right) \\
& -M^{4} k \beta_{1} \beta_{2} d_{v}^{2} N_{h}^{4} N_{v} c \lambda_{h}\left(u+d_{v}\right)-M^{3} k \beta_{1} \beta_{2} d_{v}^{2} N_{h}^{5} N_{v} \lambda_{h}^{2}\left(u+d_{v}\right), \\
C= & \left(M N_{v} \gamma+M N_{v} \rho+\lambda_{h} N_{v} N_{h}\right)\left(M^{2} k c+M k \lambda_{h} N_{h}+M c \lambda_{h} N_{h}+\lambda_{h}^{2} N_{h}^{2}\right) \\
& \left(d_{v} N_{h}^{3} N_{v} \lambda_{h}\right)-M^{3} k \beta_{1} \beta_{2} d_{v}^{2} N_{h}^{5} N_{v} \lambda_{h}^{2}\left(u+d_{v}\right)\left[Z R_{0}^{2}+1\right],
\end{aligned}
$$

where

$$
Z=\frac{N_{v} d_{v}^{2}\left(u+d_{v}\right)(\rho+\gamma)}{\beta_{1} \beta_{2} \lambda_{h}^{2} \lambda_{v} u}
$$

$I_{h}^{*}=0$, corresponds to disease free equilibrium point (DFE) which has been earlier established and $F\left(I_{h}^{*}\right)=0$ corresponds to a particular situation when the disease persists (endemic). In the case of backward bifurcation, we have multiple endemic equilibrium must exist. This implies that the equation $F\left(I_{h}^{*}\right)=0$ indicates that there are three cases we have to consider for $F\left(I_{h}^{*}\right)=0$ depending on the signs of $B$ and $C$ since $A$ is always positive.

That is;

1. If $B<0$ and $C=0$ or $B^{2}-4 A C=0$, then the equation $I_{h}^{*}=0$ and $F\left(I_{h}^{*}\right)=0$ has a unique endemic equilibrium point (one positive root) and no backward bifurcation is possible. 
2. If $C>0, B>0$ and $B^{2}-4 A C=0$, then the equation $I_{h}^{*}=0$ and $F\left(I_{h}^{*}\right)=0$ has two endemic equilibria i.e, two possible roots, and therefore there is possibility of backward bifurcation to occur.

3. Otherwise, there is none.

It is very important to note that $C$ is always positive if $R_{0}<1$ and negative if $R_{0}>1$. Hence our explanation results to the following theorem:

Theorem 5. The malaria model, we have,

(i) Exactly one unique endemic equilibrium if $C<0 \Leftrightarrow R_{0}>1$

(ii) Exactly one unique endemic equilibrium if $B<0$ and $C=0$ or $B^{2}-$ $4 A C=0$

(iii) Exactly two endemic equilibrium if $C>0, B<0$ and $B^{c}-4 A C>0$

(iv) None otherwise.

Theorem 6. The endemic equilibrium point (EE) exists iff $R_{0}>1$.

Proof. Since $A$ is positive, that is $A>0$, then $I_{h}^{*}=\frac{-B \pm \sqrt{B^{2}-4 A C}}{2 A}>0$ provided $C>0$. From the theorem above, (i), using the properties of quadratic equation, the last condition is equal to $R_{0}>1$. The part (iii), we have the possibility of backward bifurcation if we set the discriminant $B^{2}-4 A C=0$ and solve for the critical value of $R_{0}^{2}>1$. We find out that

$$
R_{0}^{2 c}=\frac{B^{2}}{4 A M^{3} k \beta_{1} \beta_{2} d_{v}^{2} N_{h}^{5} N_{v} \lambda_{h}\left(u+d_{v}\right) Z}-\frac{4 A\left[\left(M N_{v} \gamma+\right.\right.}{4 A M^{3} k \beta_{1} \beta_{2} d_{v}^{2} N_{h}^{5} N_{v} \lambda_{h}\left(u+d_{v}\right) Z}+\frac{1}{Z} .
$$

From the above, we resolved that the backward bifurcation can occur for values of $R_{0}^{c}$ lying in the range $R_{0}^{2 c}<\frac{1}{Z}<1$. Therefore $R_{0}^{2 c}<\frac{1}{Z}<1$, the Malaria Model (1) features backward bifurcation. Thus, the endemic equilibrium point (EE) exists iff $R_{0}>1$.

Theorem 7. If $R_{0}>1$, the endemic equilibrium $P_{1}$ of the model (1) is globally asymptotically stable (GAS) in $\Omega$.

Proof. We therefore, define a new dependent variable, a Lyapunov function of the form

$$
\begin{aligned}
L(t)= & \left(S_{h}-S_{h}^{*}-S_{h}^{*} \log _{e}\left(\frac{S_{h}}{S_{n}^{n}}\right)\right)+\left(E_{h}-E_{h}^{*}-E_{h}^{*} \log _{e}\left(\frac{E_{h}}{E_{n}}\right)\right)+\left(I_{h}-I_{h}^{*}-I_{h}^{*} \log _{e}\left(\frac{I_{h}}{I_{\hbar}}\right)\right) \\
& +\left(R_{h}-R_{h}^{*}-R_{h}^{*} \log _{e}\left(\frac{R_{h}}{R_{h}^{*}}\right)\right)+\left(S_{v}-S_{v}^{*}-S_{v}^{*} \log _{e}\left(\frac{S_{v}}{S_{h}^{*}}\right)\right)+\left(E_{v}-E_{v}^{*}-E_{v}^{*} \log _{e}\left(\frac{E_{v}}{E_{v}^{*}}\right)\right) \\
& +\left(I_{v}-I_{v}^{*}-I_{v}^{*} \log _{e}\left(\frac{I v}{I_{v}^{*}}\right)\right) .
\end{aligned}
$$


This implies that;

$$
\begin{aligned}
& \frac{d L}{d t}=\left(\frac{S_{h}-S_{h}^{*}}{S_{h}}\right) \frac{d S_{h}}{d t}+\left(\frac{E_{h}-E_{h}^{*}}{E_{h}}\right) \frac{d E_{h}}{d t}+\left(\frac{I_{h}-I_{h}^{*}}{I_{h}}\right) \frac{d I_{h}}{d t} \\
& +\left(\frac{R_{h}-R_{h}^{*}}{R_{h}}\right) \frac{d R_{h}}{d t} \\
& +\left(\frac{S_{v}-S_{v}^{*}}{S_{v}}\right) \frac{d S_{v}}{d t}+\left(\frac{E_{v}-E_{v}^{*}}{E_{v}}\right) \frac{d E_{v}}{d t}+\left(\frac{I_{v}-I_{v}^{*}}{I_{v}}\right) \frac{d I_{v}}{d t} . \\
& \frac{d L}{d t}=\left(\frac{S_{h}-S_{h}^{*}}{S_{h}}\right)\left[\lambda_{h} N_{h}-\frac{\beta_{1} S_{h} I_{v}}{N_{v}}+C R_{h}-\frac{\lambda_{h} S_{h} N_{h}}{M}\right] \\
& +\left(\frac{E_{h}-E_{h}^{*}}{E_{h}}\right)\left[\frac{\beta_{1} S_{h} I_{v}}{N_{v}}-k E_{h}-\frac{\lambda_{h} E_{h} N_{h}}{M}\right] \\
& +\left(\frac{I_{h}-I_{h}^{*}}{I_{h}}\right)\left[k E_{h}-\gamma I_{h}-\rho I_{h}-\frac{\lambda_{h} I_{h} N_{h}}{M}\right]+\left(\frac{R_{h}-R_{h}^{*}}{R_{h}}\right)\left[\gamma I_{h}-c R_{h}-\frac{\lambda_{h} R_{h} N_{h}}{M}\right] \\
& +\left(\frac{S_{v}-S_{v}^{*}}{S_{v}}\right)\left[\lambda_{v}-\frac{\beta_{2} S_{v} I_{h}}{N_{h}}-d_{v} S_{v}\right]+\left(\frac{E_{v}-E_{v}^{*}}{E_{v}}\right)\left[\frac{\beta_{2} S_{v} I_{h}}{N_{h}}-\left(u+d_{v}\right) E_{v}\right] \\
& +\left(\frac{I_{v}-I_{v}^{*}}{I_{v}}\right)\left[u E_{v}-d_{v} I_{v}\right] \text {. } \\
& \frac{d L}{d t}=\left(\frac{S_{h}-S_{h}^{*}}{S_{h}}\right)\left[\lambda_{h} N_{h}-\frac{\beta_{1}\left(S_{h}-S_{h}^{*}\right)\left(I_{v}-I_{v}^{*}\right)}{N_{v}}+C\left(R_{h}-R_{h}^{*}\right)-\frac{\lambda_{h}\left(S_{h}-S_{h}^{*}\right) N_{h}}{M}\right] \\
& +\left(\frac{E_{h}-E_{h}^{*}}{E_{h}}\right)\left[\frac{\beta_{1}\left(S_{h}-S_{h}^{*}\right)\left(I_{v}-I_{v}^{*}\right)}{N_{v}}-k\left(E_{h}-E_{h}^{*}\right)-\frac{\lambda_{h}\left(E_{h}-E_{h}^{*}\right) N_{h}}{M}\right] \\
& +\left(\frac{I_{h}-I_{h}^{*}}{I_{h}}\right)\left[k\left(E_{h}-E_{h}^{*}\right)-\gamma\left(I_{h}-I_{h}^{*}\right)-\rho\left(I_{h}-I_{h}^{*}\right)-\frac{\lambda_{h}\left(I_{h}-I_{h}^{*}\right) N_{h}}{M}\right] \\
& +\left(\frac{R_{h}-R_{h}^{*}}{R_{h}}\right)\left[\gamma\left(I_{h}-I_{h}^{*}\right)-c\left(R_{h}-R_{h}^{*}\right)-\frac{\lambda_{h}\left(R_{h}-R_{h}^{*}\right) N_{h}}{M}\right]+\left(\frac{S_{v}-S_{v}^{*}}{S_{v}}\right) \\
& {\left[\lambda_{v}-\frac{\beta_{2}\left(S_{v}-S_{v}^{*}\right)\left(I_{h}-I_{h}^{*}\right)}{N_{h}}-d_{v}\left(S_{v}-S_{v}^{*}\right)\right]+\left(\frac{E_{v}-E_{v}^{*}}{E_{v}}\right)\left[\frac{\beta_{2}\left(S_{v}-S_{v}^{*}\right)\left(I_{h}-I_{h}^{*}\right)}{N_{h}}\right.} \\
& \left.-\left(u+d_{v}\right)\left(E_{v}-E_{v}^{*}\right)\right]+\left(\frac{I_{v}-I_{v}^{*}}{I_{v}}\right)\left[u\left(E_{v}-E_{v}^{*}\right)-d_{v}\left(I_{v}-I_{v}^{*}\right)\right] . \\
& \frac{d L}{d t}=\frac{\left(S_{h}-S_{h}^{*}\right)^{2}}{S_{h}}\left[-\frac{\beta_{1}\left(I_{v}-I_{v}^{*}\right)}{N_{v}}-\frac{\lambda_{h} N_{h}}{M}\right]+\frac{\left(E_{h}-E_{h}^{*}\right)^{2}}{E_{h}}\left[-k-\frac{\lambda_{h} N_{h}}{M}\right] \\
& +\frac{\left(I_{h}-I_{h}^{*}\right)^{2}}{I_{h}}\left[-\gamma-\rho-\frac{\lambda_{h} N_{h}}{M}\right]+\frac{\left(R_{h}-R_{h}^{*}\right)^{2}}{R_{h}}\left[-c-\frac{\lambda_{h} N_{h}}{M}\right] \\
& +\frac{\left(S_{v}-S_{v}^{*}\right)^{2}}{S_{v}}\left[\frac{\beta_{2}\left(I_{h}-I_{h}^{*}\right)}{N_{h}}-d_{v}\right]+\frac{\left(E_{v}-E_{v}^{*}\right)^{2}}{E_{v}}\left[-\left(u+d_{v}\right)\right] \\
& +\frac{\left(I_{v}-I_{v}^{*}\right)^{2}}{I_{v}}\left[-d_{v}\right]+\left(\frac{S_{h}-S_{h}^{*}}{S_{h}}\right)\left(\lambda_{h} N_{h}\right)+\left(\frac{S_{h}-S_{h}^{*}}{S_{h}}\right)\left(c R_{h}-c R_{h}^{*}\right) \\
& +\left(\frac{E_{h}-E_{h}^{*}}{E_{h}}\right)\left[\frac{\left(\beta_{1} S_{h}-\beta_{1} S_{h}^{*}\right)\left(I_{v}-I_{v}^{*}\right)}{N_{v}}\right]+\left(\frac{I_{h}-I_{h}^{*}}{I_{h}}\right)\left[k E_{h}-k E_{h}^{*}\right] \\
& +\left(\frac{R_{h}-R_{h}^{*}}{R_{h}}\right)\left[\gamma I_{h}-\gamma I_{h}^{*}\right]+\left(\frac{S_{v}-S_{v}^{*}}{S_{v}}\right)\left[\lambda_{v}\right]+\left(\frac{E_{v}-E_{v}^{*}}{E_{v}}\right)\left[\frac{\beta_{2} S_{v}-\beta S_{v}^{*}\left(I_{h}-I_{h}^{*}\right)}{N_{h}}\right] \\
& +\left(\frac{I_{v}-I_{v}^{*}}{I_{v}}\right)\left[u E_{v}-E_{v}^{*}\right] \text {. }
\end{aligned}
$$




$$
\begin{aligned}
\frac{d L}{d t}= & \frac{\beta_{1} I_{v}^{*}\left(S_{h}-S_{h}^{*}\right)^{2}}{N_{v} S_{h}}+\frac{\left(S_{v}-S_{v}^{*}\right)^{2} \beta_{2} I_{h}}{S_{v}}+\frac{\left(S_{h}-S_{h}^{*}\right)\left(\lambda_{h} N_{h}\right)}{S_{h}} \\
& +\frac{C R_{h}\left(S_{h}-S_{h}^{*}\right)}{S_{h}}+\frac{\beta_{1} S_{h} I_{v}\left(E_{h}-E_{h}^{*}\right)}{E_{h} S_{h}^{*} I_{v}^{*}\left(E_{h}-E_{h}^{*}\right)} E_{h} \\
& k E_{h} \frac{\left(I_{h}-I_{h}^{*}\right)}{I_{h}}+\frac{\gamma I_{h}\left(R_{h}-E_{h}^{*}\right)}{R_{h}}+\frac{\lambda_{v}\left(S_{v}-S_{v}^{*}\right)}{S_{v}}+\frac{\beta_{2} S_{v}\left(E_{v}-E_{v}^{*}\right)}{N_{h} E_{v}} \\
& +\frac{\beta S_{v}^{*} I_{h}^{*}}{N_{h}} \frac{\left(E_{v}-E_{v}^{*}\right)}{E_{v}}+u E_{v} \frac{\left(I_{v}-I_{v}^{*}\right)}{I_{v}} \\
& -\frac{\beta_{1} I_{v}^{*}}{N_{v}} \frac{\left(S_{h}-S_{h}^{*}\right)^{2}}{S_{h}}-\frac{\lambda_{h} N_{h}}{M} \frac{\left(S_{h}-S_{h}^{*}\right)^{2}}{S_{h}}-k \frac{\left(E_{h}-E_{h}^{*}\right)^{2}}{E_{h}}-\frac{\lambda_{h} N_{h}}{M_{h}} \frac{\left(E_{h}-E_{h}^{*}\right)}{E_{h}} \\
& -\gamma \frac{\left(I_{h}-I_{h}^{*}\right)^{2}}{I_{h}}-\rho \frac{\left(I_{h}-I_{h}^{*}\right)^{2}}{I_{h}}-\frac{\lambda_{h} N_{h}}{I_{h}} \frac{\left(I_{h}-I_{h}^{*}\right)^{2}}{I_{h}}-c \frac{\left(R_{h}-R_{h}^{*}\right)^{2}}{R_{h}} \\
& -\frac{\lambda_{h} N_{h}}{M} \frac{\left(R_{h}-R_{h}^{*}\right)^{2}}{R_{h}}-\frac{\beta_{2} I_{h}^{*}}{N_{h}} \frac{\left(S_{v}-S_{v}^{*}\right)^{2}}{S_{v}}-d_{v} \frac{\left(S_{v}-S_{v}^{*}\right)}{S_{v}}-\left(u+d_{v}\right) \frac{\left(E_{v}-E_{v}^{*}\right)^{2}}{E_{v}} \\
& -d_{v} \frac{\left(I_{v}-I_{v}^{*}\right)^{2}}{I_{v}}-c R_{h}^{*} \frac{\left(S_{h}-S_{h}^{*}\right)}{S_{h}}-\frac{\beta_{1} S_{h}^{*} I_{v}}{N_{v}} \frac{\left(E_{h}-E_{h}^{*}\right)}{E_{h}}-\beta_{1} S_{h} I_{v}^{*} \frac{\left(E_{h}-E_{h}^{*}\right)}{E_{h}} \\
& -k E_{h} \frac{\left(I_{h}-I_{h}^{*}\right)}{I_{h}}-\gamma I_{h}^{*} \frac{\left(R_{h}-R_{h}^{*}\right)}{R_{h}}-\frac{\beta S_{v}^{*} I_{h}}{N_{h}} \frac{\left(E_{v}-E_{v}^{*}\right)}{E_{v}}-u E_{v}^{*} \frac{\left(I_{v}-I_{v}\right)}{I_{v}} .
\end{aligned}
$$

Hence by collecting positive terms terms together and negative terms together from the above equation. We arrive at

$$
\frac{d L}{d t}=A-B
$$

where

$$
\begin{aligned}
A= & \frac{\beta_{1} I_{v}^{*}}{N_{v}} \frac{\left(S_{h}-S_{h}^{*}\right)^{2}}{S_{h}}+\frac{\left(S_{v}-S_{v}^{*}\right)^{2} \beta_{2} I_{h}}{S_{v}}+\frac{\lambda_{h} N_{h}\left(S_{h}-S_{h}^{*}\right)}{S_{h}} \\
& +c \frac{R_{h}\left(S_{h}-S_{h}^{*}\right)}{S_{h}}+\frac{\beta_{1} S_{h} I_{v}\left(E_{h}-E_{h}^{*}\right)}{E_{h}}+\frac{\beta_{1} S_{h}^{*} I_{v}^{*}}{N_{v}} \frac{\left(E_{h}-E_{h}^{*}\right)}{E_{h}} \\
& +\frac{k E_{h}\left(I_{h}-I_{h}^{*}\right)}{I_{h}}+\frac{\gamma I_{h}\left(R_{h}-R_{h}^{*}\right)}{R_{h}}+\frac{\lambda_{v}\left(S_{v}-S_{v}^{*}\right)}{S_{v}}+\frac{\beta_{2} S_{v}\left(E_{v}-E_{v}^{*}\right)}{N_{h} E_{v}} \\
& \quad+\frac{\beta S_{v}^{*} I_{h}^{*}}{N_{h}}\left(\frac{E_{v}-E_{v}^{a} s t}{E_{v}}\right)+\frac{u E_{v}\left(I_{v}-I_{v}^{*}\right)}{I_{v}} . \\
B= & \frac{\beta_{1} I_{v}^{*}}{N_{v}} \frac{\left(S_{h}-S_{h}^{*}\right)^{2}}{S_{h}}+\frac{\lambda_{h} N_{h}}{M} \frac{\left(S_{h}-S_{h}^{*}\right)^{2}}{S_{h}}+\frac{k\left(E_{h}-E_{h}^{*}\right)^{2}}{E_{h}}+\frac{\lambda_{h} N_{h}}{M_{h}} \frac{\left(E_{h}-E_{h}^{*}\right)}{E_{h}} \\
+ & \frac{\gamma\left(I_{h}-I_{h}^{*}\right)^{2}}{I_{h}}+\frac{\rho\left(I_{h}-I_{h}^{*}\right)^{2}}{I_{h}}-\frac{\lambda_{h} N_{h}}{M_{h}} \frac{\left(I_{h}-I_{h}^{*}\right)^{2}}{I_{h}}+\frac{c\left(R_{h}-R_{h}^{*}\right)^{2}}{R_{h}} \\
+ & \frac{\lambda_{h} N_{h}\left(R_{h}-R_{h}^{*}\right)^{2}}{M_{2}}+\frac{\beta_{2} I_{h}^{*}}{N_{h}} \frac{\left(S_{v}-S_{v}^{*}\right)^{2}}{S_{v}}+\frac{d_{v}\left(S_{v}-S_{v}^{*}\right)^{2}}{S_{v}}+\frac{\left(u+d_{v}\right)\left(E_{v}-E_{v}^{*}\right)^{2}}{E_{v}} \\
+ & \frac{d_{v}\left(I_{v}-I_{v}^{*}\right)^{2}}{I_{v}}+\frac{c R_{h}^{*}\left(S_{h}-S_{h}^{*}\right)}{S_{h}}-\frac{\beta_{1} S_{h}^{*} I_{v}}{N_{v}}\left(\frac{E_{h}-E_{h}^{*}}{E_{h}}\right)+\frac{\beta_{1} S_{h} I_{v}^{*}}{N_{v}}\left(\frac{E_{h}-E_{h}^{*}}{E_{h}}\right) \\
+ & \frac{k E_{h}\left(I_{h}-I_{h}^{*}\right)}{I_{h}}+\frac{\gamma I_{h}^{*}\left(R_{h}-R_{h}^{*}\right)}{R_{h}}+\frac{\beta S_{v}^{*} I_{h}}{N_{h}}\left(\frac{E_{v}-E_{v}^{*}}{E_{v}}\right)+u E_{v}\left(\frac{I_{v}-I_{v}^{*}}{I_{v}}\right) .
\end{aligned}
$$

Thus if $A<B$, then we obtain that $d L d t \leq 0$; we note that $\frac{d L}{d t}=0$ if and only if $S_{h}=S_{h}^{*}, E_{h}=E_{h}^{*} ; I_{h}=I_{h}^{*} ; R_{h}=R_{h}^{*} ; S_{v}=S_{v}^{*} ; E_{v}=E_{v}^{*} ; I_{v}=I_{v}^{*}$. Therefore, 
the largest compact, invariant set in $\left[\left(S_{h}^{*}, E_{h}^{*}, I_{h}^{*}, R_{h}^{*}, S_{v}^{*}, E_{v}^{*}, I_{v}^{*}\right) \in \Omega: \frac{d L}{d t}=0\right]$ is the singleton set $P_{1}$ where $P_{1}$ is the endemic equilibrium of the system (1). By Lasalle's invariant principles it implies that $P_{1}$ is Globally Asymptotically stable in $\Omega$ if $A<B$.

\section{Numerical Simulation and Graphical Illustration of the Malaria Transmission Model}

We present the numerical solution to the optimality system Equation (4.24) to (4.31) using MATLAB with the following realistic weight factors, initial state variables and parameter values: initial state variables $S_{h}(0)=8000, E_{h}(0)=$ $2000, I_{h}(0)=5000, R_{h}(0)=3000, S_{v}(0)=700, E_{v}(0)=500, I_{v}(0)=400$ and parameter values $b_{v}=1000, b_{h}=100, \psi=0.05, \beta_{1}=0.8333, \beta_{2}=0.09, d_{h}=$ $0.00004, \alpha_{h}=0.0588, \alpha_{v}=0.0556, \varepsilon_{v}=0.2, \phi=0.502, \varepsilon_{h}=0.2, \theta=$ 0.005 for which the reproduction number $R_{0}=0.0000753$ in the presence of combined intervention strategies. We note that the parameter values above were estimated such that the total population never goes into extinction.

\section{Discussion}

In this paper, we propose a mathematical model that studied the dynamics of transmission of human-vector diseases called malaria.Our model incorporates the logistic growth function in the human compartment and take into consideration the probability that a proportion of infected mosquitoes may have contact with some proportion susceptible human and a fraction of infected human may have successful contact with some proportion of infected mosquitoes. This model is analyzed for the existence of disease-free and endemic equilibrium points.It is found that due to the presence of the logistic growth function in the model, the growth of the human population compartment slows down(due to $\frac{-N_{h}^{2}}{M}$ ) which model the internal competition in the population for vital resources such as space, food etc which is called intra-specific competition. The introduction of this internal competition model makes the population of human to possess a different behaviour different from a normal dynamics.. We realized that the model still have some limitations due to some other factors that where not incorporated. We hope to extend this work by incorporating other parameters (like introducing some control measures) and try to see how these will influence the dynamics of transmission in the presence of the logistic growth 


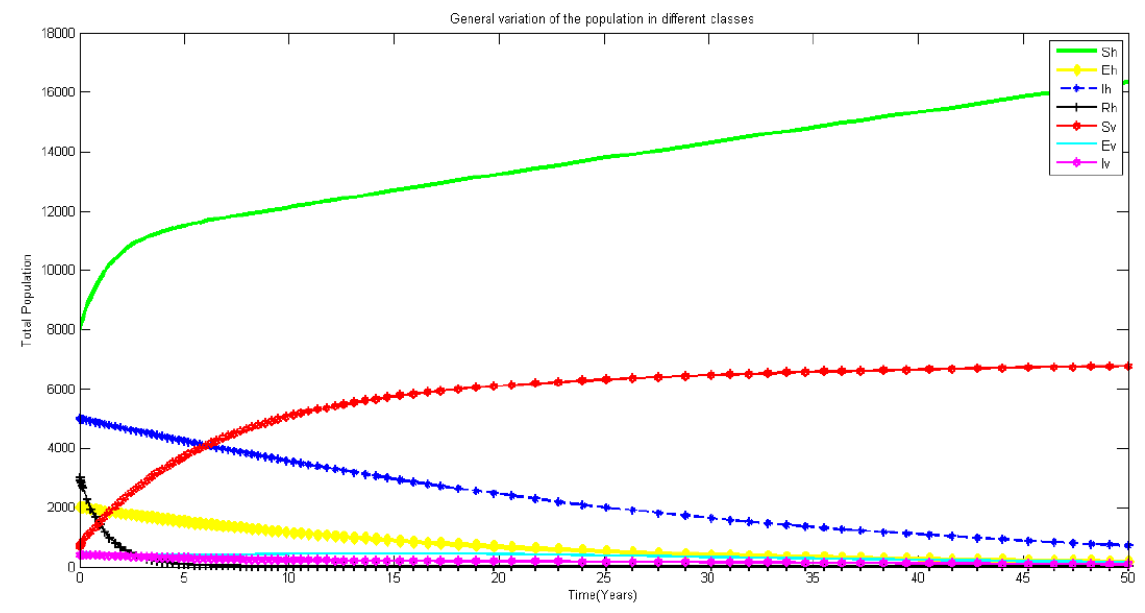

Figure 1: In the figure (5.1) above, it is found that, susceptible human population increases with time even because of natural wanning immunity that makes some fractions of recovered individuals returns to susceptible class. The exposed and infected human population also decreases with time due to the extrinsic growth rate in human population.It is observed that the recovered population keep on decreasing as time increases and then finally enters into extinction. The population of the susceptible mosquitoes increases as time increases and maintain a steady state on its carrying capacity, while the population of the exposed and infected mosquitoes decreases with time. The plot actually shows that the susceptible human population keep increasing which means that the disease will remain endemic in the population.

functions.It should be noted that due to lack of space especially in the urban area and competition for food there has been many cases of malaria diseases. These factors will increase the rate at which the disease are contracted and thereby increase the rate of infection in human and vectors.

\section{References}

[1] R. Aguas, M.U. Ferreira, M.G.M. Gomes, Modeling the effects of relapse in the transmission dynamics of malaria parasites, Journal of Parasitology Research 2012 (2012) 8. 
(c) Variation of infective human population for different values of beta1

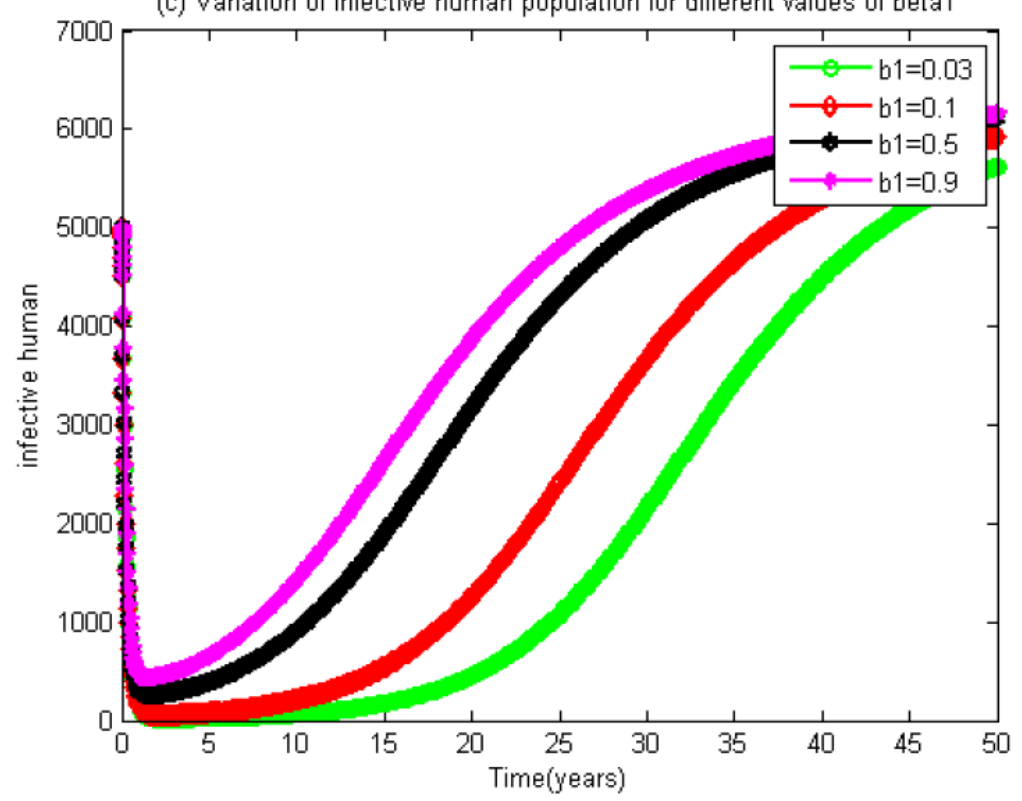

Figure 2: In the figure (5.2), shows that the infected human population increases with increasing transmission rate.

[2] R.M. Anderson, R.M. May, Infectious Diseases of Humans: Dynamics and Control, Oxford University Press, Oxford, (1991).

[3] Cai Li-Ming, Abid Ali Lashari, Il Hyo Jung, Kazeem Oare Okosun, and Young Il Seo, "Mathematical Analysis of a Malaria Model with Partial Immunity to Reinfection"Hindawi Publishing Corporation Abstract and Applied Analysis Volume 2013, Article ID 405258, 17 pages http://dx.doi.org/10.1155/2013/405258

[4] Chitnis Nakul, J. M. Cushing, J. M. Hyman, Bifurcation analysis of a mathematical model for malaria transmission, SIAM J. APPL. MATH, Volume 67, Society for Industrial and Applied Mathematics, 2006.

[5] Chiyaka C., Garira W., Dube S., Effects of treatment and drug resistance on the transmission dynamics of malaria in endemic areas, Theor. Pop. Biol. 75, pp 14-29 (2009).

[6] Chiyaka C., Tchuenche J. M. Garira W. and Dube S., A mathematical 


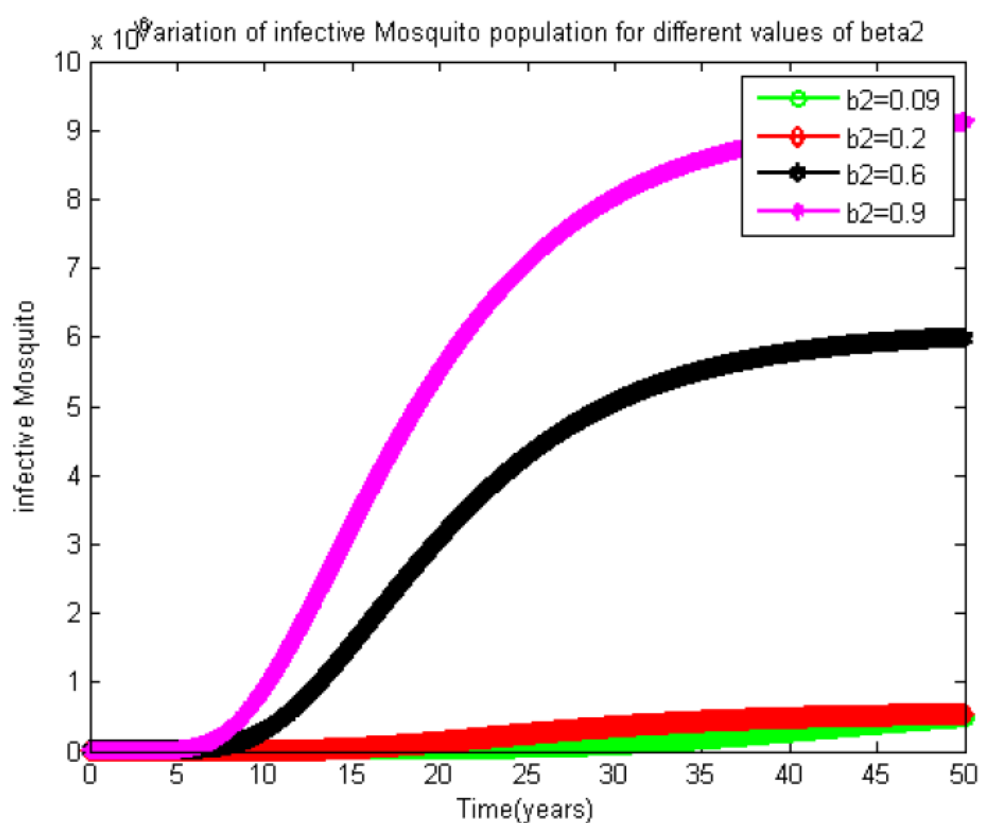

Figure 3: In the figure (5.3) above, shows that the infected mosquito population also increases with increasing transmission rate.

analysis of the effects of control strategies on the transmission dynamics of malaria, App. Math. Comp. 195, pp 641-662 (2008).

[7] C. Chiyaka, W. Garira, S. Dube, Transmission model of endemic human malaria in a partially immune population, Elsevier Mathematical and Computer Modelling, 2007.

[8] Dietz K, L. Molineaux, A. Thomas, A malaria model tested in Africa Savannah, Bull, WHO, 1974.

[9] Hyun M Yang, Malaria transmission model for different levels of acquired immunity and temperature-dependent parameters (vector), Journal of Public Health, Volume 34, 2000

[10] Isao K, Akira S, Motoyoshi M (2004). Combining zooprophylaxis and insecticide spraying: a malaria-control strategy limiting the development of insecticide resistance in vector mosquitoes. Proc. R. Soc. Lond., 271: 301 - 309 . 
[11] Jia, L.,2008.A malaria model with partial immunity in humans.Mathematical Biosciences and Engineering5(4),789801.

[12] Koella J. C., Antia R., Epidemiological models for the spread of antimalarial resistance, Malaria J. 2:pp 3 (2003).

[13] Labadin J., C. Kon M.L, S.F.S. Juan, "Deterministic Malaria Transmission model with Acquired Immunity", Proceedings of the World Congress on Engineering and Computer Science 2009 Vol II WCECS 2009, October 20-22, 2009, San Francisco, USA.

[14] Lopez-velez R., H. Huerga, M.C. Turrientes, Infectious diseases in immigrants from the perspective of a tropical medicine referral unit, Am. J. Trop. Med. Hyg. 69 (1) (2003) 115121.

[15] Macdonald G, The epidemiology and control of malaria, Oxford: Oxford University Press, 1957.

[16] Makinde O.D, K.O Okosun. "Impact of Chemotherapy on Optimal Control of malaria disease with infected immigrants" Biosystems 104(2012) 32-41.

[17] Martens P., L. Hall, Malaria on the move: Human population movement and malaria transmission, Emerging Infect. Dis. 6 (2) (2000) 103109.

[18] Mukandavire Z., Garira W., Tchuenche J.M., "Modelling effects of public health educational campaigns on HIV/AIDS transmission dynamics", Applied Mathematical Modelling 33 (2009) 20842095.

[19] Mwasa A., Tchuenche J.M., " Mathematical analysis of a cholera model with public health interventions", Biosystems 105(2011) 190-200.

[20] Ngwa G.A.,Shu W.S., A mathematical model for the endemic malaria with variable human and mosquito populations, Math. Comput. Modeling, 2000, pp.747-763

[21] Ross R, The prevention of malaria, London: John Murray, 1910.

[22] K. Singh, W.C. Wester, M. Gordon, G.M. Trenholme, Problems in the therapy for imported malaria in the United States, Arch. Intern. Med. 163 (17) (2003) 20272030.

[23] Tumwiine J., J.Y.T Mugisha, L.S. Luboobi," J. Math. Anal.Appl. 361(2010) 139-149. 
[24] Tumwiine J., J.Y.T. Mugisha and L.S. Luboobi. Threshold and stability results for a malaria model in a population with protective intervention among high-risk groups. Math. Model. Anal., 13(3):443460, 2008. (doi:10.3846/1392-6292.2008.13.443-460)

[25] Tumwiine J., L.S. Luboobi, J.Y.T. Mugisha, Modelling the effect of treatment and mosquitoes control on malaria transmission, International Journal of Management and Systems 21 (2) (2005) 107124.

[26] Tumwiine J., J.Y.T. Mugisha, L.S. Luboobi, A mathematical model for the dynamics of malaria in a human host and mosquito vector with temporary immunity, Elsevier Applied Mathematics and Computation, 2007.

[27] White M, Griffin J, Churcher T, Ferguson N, Basanez MG, Ghani A: Modelling the impact of vector control interventions on Anopheles gambiae population dynamics. Parasit Vectors 2011, 4:153.

[28] Wilson M.E., Infectious diseases: An ecological perspective, Br. Med. J. 311 (1998) 16811684. 
